\title{
A giant skin tag of the scrotum and verruca anogenitalis
}

\author{
Mehmet Eren Yuksel'1, Funda Tamer ${ }^{2}$
}

${ }^{1}$ Department of General Surgery, Devrek State Hospital, Zonguldak, Turkey, ${ }^{2}$ Department of Dermatology, Medical Park Hospital, Ankara, Turkey

Corresponding author: Dr. Funda Tamer, E-mail: fundatmr@yahoo.com

Sir,

A 65-year-old Caucasian male presented with a $6 \times 4 \times 3 \mathrm{~cm}$ mass on the scrotal skin. The patient admitted that the lesion had been present for about ten years and it increased in size gradually. The past medical history was unremarkable. Physical examination revealed a hyperpigmented, erythematous, polypoid mass hanging from the left scrotal skin with a peduncle. The lesion was soft and nontender to palpation. In addition, there was a hyperkeratotic, skin colored, small papule on the left scrotum above the giant polypoid mass measuring $2 \times 3 \times 4 \mathrm{~mm}$ in size (Fig. 1). The patient stated that the small lesion appeared for about two months ago. Both of the lesions were removed surgically under local anesthesia to reach a definitive diagnosis (Figs. 2a and 2b). Histopathological examination of the polypoid mass and the small papule revealed a skin tag and verruca anogenitalis, respectively.

A skin tag, also known as acrochordon or fibroepithelial polyp, is a common benign mesenchymal tumor. It usually presents as a small, pedunculated polyp in intertriginous areas such as neck, axilla, groin and face. Rarely, skin tags may appear in the respiratory tract, orophyarynx, female and male genitalia $[1,2]$. Skin tags affect $46 \%$ of the general population. Moreover, they are usually seen in elderly women during perimenopausal and postmenopausal period [2]. Genetic predisposition, obesity, impaired glucose tolerance, diabetes mellitus and hyperlipidemia may be the causative factors [1-3]. Furthermore, human papilloma virus (HPV) infection may play role in the etiology of skin tags [3].

Giant skin tags are rare, and a few giant skin tag cases have been reported previously to the best of

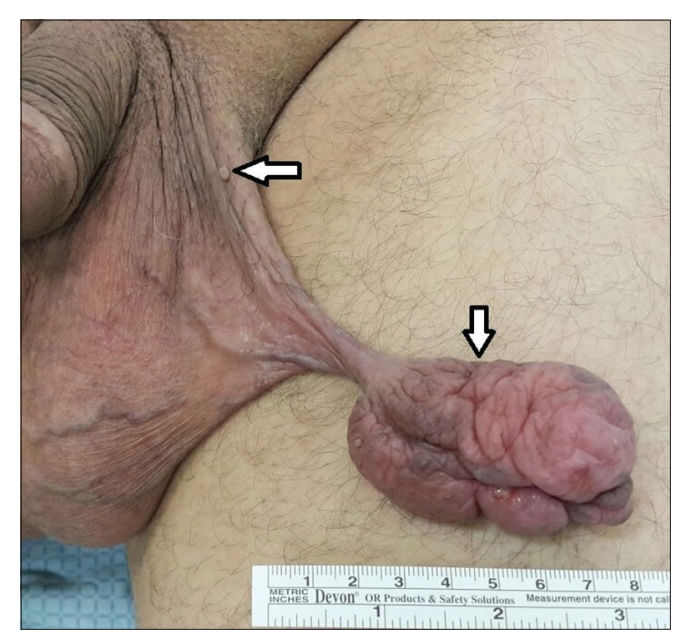

Figure 1: A hyperpigmented, erythematous, polypoid mass measuring $6 \times 4 \times 3 \mathrm{~cm}$ in size and a hyperkeratotic, skin colored, small papule on the left scrotal skin (white arrows).

our knowledge. Ghosh et al. reported four cases with giant skin tags on unusual localizations including nipple $(3 \times 2.5 \mathrm{~cm})$, eyelid $(0.5 \times 2 \mathrm{~cm})$ and right thigh $(2.5 \times 2.5 \mathrm{~cm})$ [2]. Yan et al. reported a 62-year-old patient with a giant skin tag measuring $7 \times 5 \times 3 \mathrm{~cm}$ on penis with verrucous appearance [1]. Ilango et al. described a giant skin tag measuring $12 \times 8 \mathrm{~cm}$ on the back with variable pigmentation [4]. Colak et al. presented a female with a giant skin tag measuring $18 \times 9 \times 3 \mathrm{~cm}$ on the perineum [5]. Canalizo-Almedia et al. reported two patients with giant skin tags on their labia majora measuring $15 \mathrm{~cm}$ and $30 \mathrm{~cm}$ in diameter [3].

The diagnosis of a skin tag is usually made by its clinical features [3]. Buschke-Lowenstein tumor, pedunculated seborrheic keratosis and neurofibromatosis should be included in the differential diagnosis. Electrocauterization and cryotherapy are the treatment

\footnotetext{
How to cite this article: Yuksel ME, Tamer F. A giant skin tag of the scrotum and verruca anogenitalis. Our Dermatol Online. 2017;8(2):239-240. 
www.odermatol.com

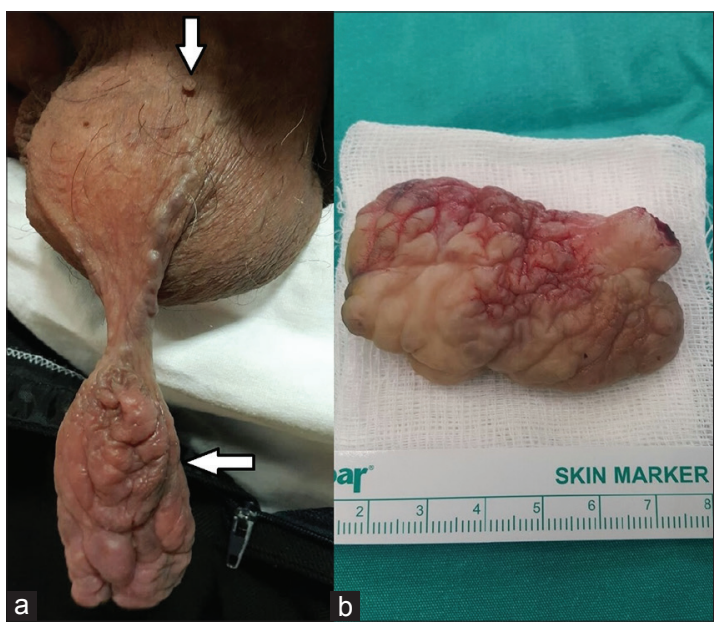

Figure 2: a) A polypoid mass hanging from the left scrotal skin with a peduncle and a hyperkeratotic, small papule above the giant lesion (white arrows) b) The specimen after surgical excision.

options. Surgical removal is recommended for large lesions, however recurrence may occur $[1,2]$.

In conclusion, giant skin tags have been reported on labium majus, penis and perineum previously. However, a $6 \times 4 \times 3 \mathrm{~cm}$ giant skin tag on the scrotal skin is the first case report in the English medical literature to the best of our knowledge. Moreover, the patient had both a giant skin tag and verruca anogenitalis on the scrotal skin. These findings support that HPV infection may play role in the etiology of skin tags.

\section{REFERENCES}

1. Yan H, Treacy A, Yousef G, Stewart R. Giant fibroepithelial polyp of the glans penis not associated with condom-catheter use: A case report and literature review. Can Urol Assoc J. 2013;7:E621-4.

2. Ghosh SK, Bandyopadhyay D, Chatterjee G, Bar C. Giant skin tags on unusual locations. J Eur Acad Dermatol Venereol. 2009;23:233.

3. Canalizo-Almeida S, Mercadillo-Pérez P, Tirado-Sánchez A. Giant skin tags: report of two cases. Dermatol Online J. 2007;13:30.

4. Ilango N, Jacob J, Gupta AK, Choudhrie L. Acrochordon-a rare giant variant. Dermatol Surg. 2009;35:1804-5.

5. Colak E, Ikinci A, Kucuk GO, Kesmer S, Yildirim K. Giant fibroepithelial polyp of the perineum: Giant fibroepithelial polyp. Int J Surg Case Rep. 2015;17:126-7.

Copyright by Mehmet Eren Yuksel, et al. This is an open access article distributed under the terms of the Creative Commons Attribution License which permits unrestricted use, distribution, and reproduction in any medium, provided the original author and source are credited. Source of Support: Nil, Conflict of Interest: None declared. 